\title{
Biochemical Genetical Studies on the Pathway of Sulphate Assimilation in Aspergillus nidulans
}

\author{
BY T. NAKAMURA \\ Institute for Protein Research, Osaka University, Kita-Ku, Osaka, Japan
}

(Received 23 May 1961)

\section{SUMMARY}

Mutant strains of Aspergillus nidulans requiring methionine for growth were isolated and their growth responses to inorganic and organic sulphur sources studied. It is suggested that methionine is synthesized in this mould from inorganic sulphate through sulphite, thiosulphate and cysteine. The mutant strains genetically blocked in the reaction from thiosulphate to cysteine could be divided into two genetically different groups by means of the heterokaryosis test; heterokaryons formed between these two groups were able to grow on sulphate as sole sulphur source because of the syntrophic action of two different types of nuclei. It was further shown that cysteine-S-sulphonate supported good growth of one of the two groups, whereas the other group showed no growth on this compound. It was concluded that the metabolic conversion of thiosulphate to cysteine involves cysteine-S-sulphonate as an intermediate. Mutant strains responded to sulphide in the same way as to thiosulphate. From this and other evidence, it is suggested that sulphide is utilized by way of thiosulphate.

\section{INTRODUCTION}

Despite numerous studies so far reported, no conclusive scheme has yet been presented for the metabolic pathway by which inorganic sulphate is converted to sulphur-containing amino acids in micro-organisms. Recent investigations have established that the first step of this assimilation involves the activation of sulphate by means of adenosine triphosphate and subsequent reduction of the active sulphate to sulphite (Wilson \& Bandurski, 1958; Hilz \& Kittler, 1958; Hilz, Kittler \& Knape, 1959; Ragland, 1959). Three different pathways have, however, been suggested for the further fate of the sulphite sulphur.

In one of the three suggested possibilities it is postulated that sulphite directly reacts with pyruvate to form $\beta$-sulphinyl-pyruvate which is then converted to cysteine via cysteine sulphinate. This pathway has been suggested to be operative in Aspergillus nidulans (Shepherd, 1956). The formation in rabbit kidney of radioactive cysteine sulphinate from ${ }^{35} \mathrm{~S}$-labelled sulphite has also been reported (Chapeville \& Fromageot, 1954).

In the second pathway, first suggested on the basis of evidence obtained with Escherichia coli, it is assumed that sulphite sulphur is reduced to the sulphide state before its introduction into the carbon chain of cysteine (Lampen, Roepke \& Jones, 1947). Evidence for this pathway seems to be given by the finding that a pyridoxal phosphate-dependent enzyme occurs in yeast and catalyses the condensation of 
hydrogen sulphide with serine (Schlossmann \& Lynen, 1957). Little is, however, known of the mechanism by which sulphite is reduced to sulphide, except for the report that this reduction can be catalysed by a very unstable enzyme system in yeast which uses reduced triphosphopyridine nucleotide as hydrogen donor (Lezius, 1959). Working with enzyme preparations from yeast, Hilz et al. (1959) excluded thiosulphate as an intermediate in this reduction process.

In contrast to the above two pathways, it is suggested as a third possibility that sulphite is converted to thiosulphate and that the sulphur-carbon linkage is formed at this stage of sulphate reduction. This pathway was proposed by Horowitz (1950), based on observations with nutritional mutants of Neurospora crassa. It has further been suggested from studies with mutant strains of Aspergillus nidulans that thiosulphate reacts with serine to give cysteine-S-sulphonate as an intermediate in the biosynthesis of cysteine (Hockenhull, 1949). Shepherd (1956) also considered this possibility, but denied the involvement of thiosulphate in the main route of sulphate assimilation.

The present work was undertaken with the hope of throwing light on the problem of sulphate assimilation by using a biochemical genetical approach. Mutant strains of Aspergillus nidulans which grew with methionine as a nutrient component were used for this purpose. The results obtained convincingly indicate that cysteine-Ssulphonate is an obligatory intermediate in sulphate assimilation in $\boldsymbol{A}$. nidulans and that thiosulphate is the form in which sulphate-sulphur combines with the carbon skeleton of cysteine in the assimilatory process. A preliminary report of this work was published elsewhere (Nakamura \& Sato, 1960).

\section{METHODS}

Organism. A strain of Aspergillus nidulans IFO 5713 was supplied from the Institute for Fermentation, Osaka.

Media. The basal sulphur-deficient medium contained per l. deionized water: 30 g. glucose; 3 g. $\mathrm{NaNO}_{3} ; 1$ g. $\mathrm{KH}_{2} \mathrm{PO}_{4} ; 0.5$ g. $\mathrm{K}_{2} \mathrm{HPO}_{4} ; 0.2 \mathrm{~g} . \mathrm{MgCl}_{2} ; 0 \cdot 1 \mathrm{~g}$. $\mathrm{CaCl}_{2} \cdot 2 \mathrm{H}_{2} \mathrm{O} ; 1 \mathrm{ml}$. trace element solution. This medium was at $\mathrm{pH} 6 \cdot 6-6 \cdot 8$ before autoclaving. The trace element solution contained (mg./l.): $\mathrm{FeCl}_{3} \cdot 6 \mathrm{H}_{2} \mathrm{O}, 1000$; $\mathrm{CuCl}_{2} \cdot 2 \mathrm{H}_{2} \mathrm{O}, 300 ; \mathrm{MnCl}_{2} \cdot 4 \mathrm{H}_{2} \mathrm{O}, 72 ;\left(\mathrm{NH}_{4}\right)_{6} \mathrm{Mo}_{7} \mathrm{O}_{24} \cdot 4 \mathrm{H}_{2} \mathrm{O}, 40 ; \mathrm{ZnCl}_{2}, 4200$. The basal medium was supplemented with various sulphur compounds (100 mg./l.) for use in nutritional tests. When thiosulphate, sulphide or cysteine-S-sulphonate was added as sulphur source, $\mathrm{KH}_{2} \mathrm{PO}_{4}$ was omitted and the amount of $\mathrm{K}_{2} \mathrm{HPO}_{4}$ was increased to $1.2 \mathrm{~g}$./l. This modification resulted in an increase in $\mathrm{pH}$ of the medium to $7 \cdot 4-7 \cdot 6$. Sulphite, thiosulphate, sulphide, cysteine-S-sulphonate and cysteine were separately sterilized by Seitz filtration and aseptically added to the steamsterilized basal medium. The basal medium supplemented with sulphate, etc., will be designated simply as 'sulphate medium', etc. When solid media were needed, $17 \mathrm{~g}$. purified agar $/ 1$. were added. Autoclaving was at $121^{\circ}$ for $15 \mathrm{~min}$.

The germination medium used for isolating mutants contained, per $800 \mathrm{ml}$.: 30 g. glucose, 3 g. $\mathrm{NaNO}_{3}, 0 \cdot 2$ g. $\mathrm{MgCl}_{2}, 0 \cdot 76$ g. $\mathrm{KCl}, 0 \cdot 01 \mathrm{~g} . \mathrm{FeCl}_{3} \cdot 6 \mathrm{H}_{2} \mathrm{O}, 0 \cdot 3 \mathrm{mg}$. $\mathrm{CuCl}_{2} \cdot 2 \mathrm{H}_{2} \mathrm{O}, 1 \cdot 0 \mathrm{~g} . \mathrm{Na}_{2} \mathrm{SO}_{4}$. In germination experiments, 8 volumes of this medium were mixed with 2 volumes of conidiospore suspension in sterilized $0 \cdot 1 \mathrm{M}$-phosphate buffer ( $\mathrm{pH} 7 \cdot 0$; containing $0 \cdot 29 \mathrm{~g} . \mathrm{KH}_{2} \mathrm{PO}_{4}, 0 \cdot 77 \mathrm{~g} . \mathrm{K}_{2} \mathrm{HPO}_{4} / 200 \mathrm{ml}$.); this mixture was at $\mathrm{pH} 6 \cdot 8-7 \cdot 0$. 
Compounds. Sodium L-cysteine-S-sulphonate was prepared by a modification (Nakamura, unpublished) of the method of Kolthoff \& Stricks (1951) and purified by chromatography on a Dowex-1 column. Choline sulphate was synthesized according to Schmidt \& Wagner (1904). Sulphate, sulphite, thiosulphate and sulphide were all used as sodium salts. The cysteine, cystine and methionine used were all L-isomers.

Isolation of mutants. Conidiospores of Aspergillus nidulans were collected from freshly grown slope cultures on the sulphate medium and suspended in $0 \cdot 1 \mathrm{M}$ potassium phosphate buffer ( $\mathrm{pH} \mathrm{7 \cdot 0)}$ ). The suspension was shaken for $2 \mathrm{hr}$. to break the spore chains and filtered through gauze into Petri dishes. The filtered spore suspension $(5 \mathrm{~mm}$. deep) was then exposed to ultraviolet radiation (10 W. Toshiba UV lamp, $100 \mathrm{~V}$.) from a distance of $40 \mathrm{~cm}$. for $8 \mathrm{~min}$. The survival rate under these conditions was $6.7 \%$. Twenty $\mathrm{ml}$. of irradiated suspension was mixed with $80 \mathrm{ml}$. germination medium (containing $\mathrm{Na}_{2} \mathrm{SO}_{4}$ as sole sulphur source) and the mixture shaken at $32^{\circ}$. After shaking for $16,20,24$ or $28 \mathrm{hr}$, , cultures were filtered through 3 to 4 sheets of gauze to remove the mycelia of wild-type strains which had grown in this medium (Fries, 1947). The filtrate from the final filtration in which spores of mutant strains had been enriched was variously diluted and plated on the methionine agar medium. The colonies thus developed were picked on to methionine agar slopes to confirm their ability to grow on this medium. They were then transferred to the sulphate agar medium and those strains which failed to grow on the latter medium were selected as auxotrophic mutants. The stability of the mutants thus isolated was confirmed by monospore isolation and serial transfer to appropriate media. In all, 694 strains which had grown on the methionine medium were subjected to the screening as described and 14 stable methionine-requiring mutants were isolated (yield, $2 \cdot 02 \%$ ).

Nutritional tests. Utilization for growth of various sulphur sources by the wildtype and mutant strains was studied as follows. Spores of each strain were separately inoculated into each of two sterile plugged tubes $(15 \times 120 \mathrm{~mm}$.) each containing $15 \mathrm{ml}$. of basal medium supplemented with the sulphur compound to be tested. The tubes were incubated at $32^{\circ}$ for 8 days and the growth examined. At least three independent sets of experiments were carried out to judge the growth response. Quantitative experiments were also made with cysteine-S-sulphonate as the sulphur source. The mycelia grown after desired periods of incubation were collected on pre-weighed sheets of filter paper, washed with water, and dried at $90^{\circ}$ for about $4 \mathrm{hr}$. The dried mycelia, together with the filter papers, were then kept overnight over $\mathrm{P}_{2} \mathrm{O}_{5}$ in vacuo and the dry weight measured, the weight of filter paper being subtracted.

Production of heterokaryons. Heterokaryosis due to the vegetative hyphal fusion between two genetically distinguished mutants of a fungus (Pontecorvo, 1947, 1953) was extensively used in the present study to investigate metabolic pathways. The method used was based on the so-called limiting nutrition technique (Pontecorvo \& Sermonti, 1954; Pontecorvo, Gloor \& Forbes, 1954). Thick conidial suspensions $\left(0.5\right.$ to $5 \times 10^{7}$ conidia $/ \mathrm{ml}$.) from the two mutants to be tested were well mixed and the mixture plated on the basal agar medium supplemented with $5 \mathrm{mg}$. (suboptimal) methionine $+100 \mathrm{mg}$. (sufficient) $\mathrm{Na}_{2} \mathrm{SO}_{4} /$ l. In certain instances, mixed point inoculation was also made with the conidial suspensions at fixed 
points on the agar medium. On incubating the mixed culture at $32^{\circ}$, the methionine suboptimally added to the medium permitted growth of both mutants and thus permitted the fusion between the homokaryotic hyphae of the component strains. When the genetic blocks in the two mutants used are located at different steps of the sole pathway from inorganic sulphate to methionine, the heterokaryon formed by the fusion must now be able to grow upon sulphate alone because of the syntrophic action of the two different types of nuclei in the heterokaryotic mycelium. When, however, the two component strains are genetically identical, such utilization of sulphate cannot be expected. The formation of heterokaryons in the mixed culture could thus be easily detected by observing the development of distinct large colonies on this medium. After incubation for 4-5 days, small mycelial tips of the heterokaryotic colonies were transferred to the sulphate medium and those which again showed good growth were selected as 'balanced' heterokaryons. In order further to confirm that the growth on sulphate alone was not due to contamination or to reversed mutation, these heterokaryons were examined by single conidium isolation. Since the conidium of Aspergillus nidulans is haploid and uninucleate, a single spore from a heterokaryon should bear the character of one or other of the component strains, except in the case of mitotic recombination. The rate of such recombination was, however, reported to be $1 / 10^{6}$ to $10^{7}$ (Pontecorvo, 1953) and thus could be neglected. Conidiospores from the heterokaryon to be tested were therefore sown (about 500 conidia per Petri dish) on both the methionine and sulphate media and incubated at $32^{\circ}$ for several days. When the sown conidia were taken from a true heterokaryon, active growth was observed only on the methionine medium and no colonies were formed on the sulphate medium. Sometimes one or two colonies were observed on the sulphate medium, but this might have been caused either by contamination of mycelial tips or by mitotic recombination. The formation of heterokaryons between two mutant strains was accepted only after these confirmations.

\section{RESULTS}

Response of mutants to various sulphur sources

In Table 1 are summarized the growth responses of the isolated 14 auxotrophic mutants (together with that of the wild-type strain) to various inorganic and organic sulphur sources. The wild-type strain utilized for growth all the sulphur compounds tested. It is clear from these results that, according to their nutritional requirements, the mutants can be classified into 4 groups which will be called groups $A, B, C$ and $D$ as indicated in Table 1. It appears that the simplest explanation for these findings as well as those reported earlier (Lederberg \& Tatum, 1946; Hockenhull, 1949) may be provided by the following scheme of sulphate assimilation:

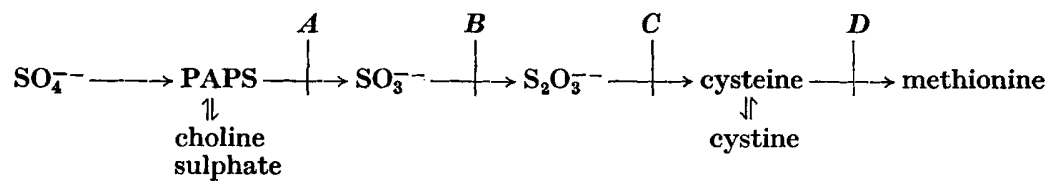

Here, the letters $A, B$, etc., indicate the sites of block in respective groups of mutants. Adenosine $3^{\prime}$-phosphate 5'-sulphato-phosphate (PAPS) is inserted in the 
scheme to show the position of choline sulphate as elucidated by recent studies (Kaji \& Gregory, 1959; Spencer \& Harada, 1960). The role of sulphide in assimilation will be discussed later.

Table 1. Growth responses of wild-type and mutant strains of Aspergillus nidulans to various sulphur sources

\begin{tabular}{|c|c|c|c|c|c|c|c|c|c|}
\hline \multirow{3}{*}{ Group } & \multirow{3}{*}{ Strain } & \multicolumn{8}{|c|}{ Sulphur sources } \\
\hline & & $\mathrm{Na}_{2} \mathrm{SO}_{4}$ & $\begin{array}{l}\text { Choline } \\
\text { sulphate }\end{array}$ & $\mathrm{Na}_{2} \mathrm{SO}_{3}$ & $\mathrm{Na}_{2} \mathrm{~S}$ & $\mathrm{Na}_{2} \mathrm{~S}_{2} \mathrm{O}_{3}$ & Cysteine & Cystine & \multirow[t]{2}{*}{$\begin{array}{c}\text { Methio } \\
\text { nine }\end{array}$} \\
\hline & & \multicolumn{7}{|c|}{ Growth response } & \\
\hline - & $\begin{array}{l}\text { Wild- } \\
\text { type }\end{array}$ & + & + & + & + & + & + & + & + \\
\hline$A$ & 954 & - & - & + & + & + & + & + & + \\
\hline$B$ & $5 \pi 4$ & - & - & - & + & + & + & + & + \\
\hline$C$ & 562 & - & - & - & - & - & + & + & + \\
\hline$C$ & 611 & - & - & - & - & - & + & + & + \\
\hline$C$ & 649 & - & - & - & - & - & + & + & + \\
\hline$C$ & 721 & - & - & - & - & - & + & + & + \\
\hline $\boldsymbol{C}$ & 793 & - & - & - & - & - & + & + & + \\
\hline$C$ & 831 & - & - & - & - & - & + & + & + \\
\hline$D$ & 519 & - & - & - & - & - & - & - & + \\
\hline$D$ & 523 & - & - & - & - & - & - & - & + \\
\hline $\boldsymbol{D}$ & 795 & - & - & - & - & - & - & - & + \\
\hline$D$ & 845 & - & - & - & - & - & - & - & + \\
\hline$D$ & 950 & - & - & - & - & - & - & - & + \\
\hline$D$ & 984 & - & - & - & - & - & - & - & + \\
\hline
\end{tabular}

\section{Thiosulphate as intermediate}

As already mentioned, it has been established that the reduction of sulphate to sulphite is an obligatory step in the microbial synthesis of sulphur-containing amino acids from sulphate. It has also been suggested that in Neurospora crassa methionine is formed from cysteine through the cystathionine pathway (Teas, Horowitz \& Fling, 1948; Fling \& Horowitz, 1951). The scheme proposed above for sulphate assimilation would, therefore, be acceptable if it were possible to show that cysteine is synthesized from sulphite via thiosulphate. A support for this may be found in Table 1 in which it is shown that all the 12 mutants which were unable to utilize thiosulphate (groups $C$ and $D$ ) also did not grow on sulphite, and hence on sulphate, as sulphur sources. Furthermore, other evidence in favour of this possibility was obtained in heterokaryosis tests between thiosulphateless (group $B$ ) and cysteineless (group $C$ ) mutants. When strain 574 belonging to group $B$ was subjected to hyphal anastomosis with 3 strains of group $C$, the formation of heterokaryons was detected in each pair; and the heterokaryons thus formed were capable of growing on sulphate as the sole source of sulphur.

These two pieces of evidence strongly suggest that thiosulphate is in fact a normal intermediate in the synthesis of cysteine from sulphate and sulphite by Aspergillus nidulans. But these results can also be equally well accounted for by assuming that the actual intermediate is not thiosulphate itself, but is a compound which can be readily derived from it. Further studies are, therefore, necessary to 
decide which is really the case; but it seems fairly certain that either thiosulphate itself or a compound in equilibrium with it (e.g. enzyme-bound thiosulphate) lies on the normal pathway of cysteine biosynthesis in Aspergillus nidulans.

\section{Incorporation of thiosulphate into cysteine}

If thiosulphate can be admitted as a normal intermediate in sulphate assimilation, it is most probable that the sulphur-carbon bond in cysteine is formed at this stage of sulphate reduction. Hockenhull (1949) suggested that in Aspergillus nidulans thiosulphate reacts with serine to form cysteine-S-sulphonate as a preparatory step in the synthesis of cysteine. The validity of this hypothesis was studied by using the 6 strains of group $C$ in which the conversion of thiosulphate to cysteine

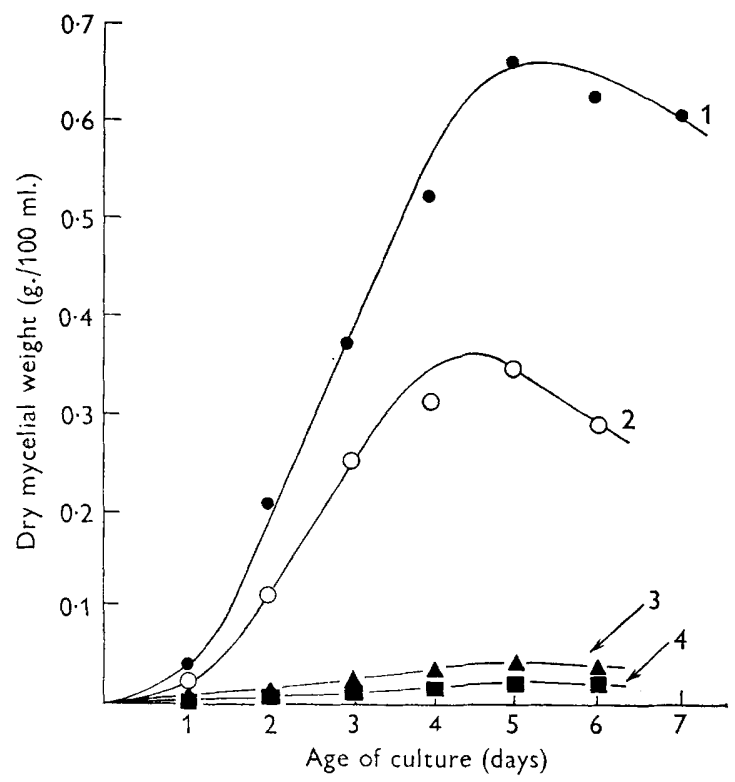

Fig. 1. Utilization of cysteine-S-sulphonate as sulphur source by several strains of Aspergillus nidulans. Experimental conditions as described in text. $1=$ wild-type strain ; 2 = strain 721 ( $C$, cysteine-S-sulphonateless) ; $3=$ strain 950 ( $D$, methionineless); $4=$ strain $793(C$, cysteineless).

Table 2. Growth of wild-type strain and group C mutants of Aspergillus nidulans on cysteine-S-sulphonate as sulphur source

Average of four independent experiments. Yield of growth is expressed in terms of dry mycelial weight after 10 days cultivation in 15 ml. medium containing $100 \mathrm{mg}$. cysteine-S-sulphonate/1.

\begin{tabular}{|c|c|c|c|c|c|c|}
\hline \multicolumn{7}{|c|}{ Strains } \\
\hline Wild-type & 793 & 562 & 611 & 649 & 721 & 831 \\
\hline \multicolumn{7}{|c|}{ Yield of mycelium (mg. dry weight) } \\
\hline
\end{tabular}

* This strain showed rather poor growth even in a methionine medium. 
is the site of genetic blocking. If this conversion actually involves cysteine-Ssulphonate as an intermediate, it might be expected that this step would be controlled by at least two genes. To examine this possibility, the 6 strains of group $C$ were subjected to an allelism test by observing the production of heterokaryons on a medium containing sufficient sulphate and suboptimal amounts of methionine. It was thus found repeatedly that one strain (strain 793) of the 6 formed heterokaryons with any of the other 5 strains (strains 562, 611, 649, 721, 831). No signs of heterokaryosis were, however, detected with any pair of the latter 5 strains, indicating their genetical identity. Having thus confirmed that the block locus in strain 793 is different from that in the other 5 strains of group $C$, the ability of these mutants to utilize cysteine-S-sulphonate as sole sulphur source was then examined. As can be seen from Table 2 and Fig. 1, strain 793 was quite unable to utilize cysteine-S-sulphonate as sulphur source, whereas the other 5 strains as well as the wild-type strain grew well on this compound. Fig. 1 also shows that a strain of group $D$ (strain 950) in which the reaction from cysteine to methionine is blocked did not utilize the sulphonate.

These results, together with those of Table 1, strongly suggest that strain 793 has a genetical block between cysteine-S-sulphonate and cysteine and that the other 5 strains of group $C$ are deficient in the enzyme responsible for the condensation of thiosulphate with a 3-carbon compound to form the sulphonate. This situation is illustrated in the following scheme:

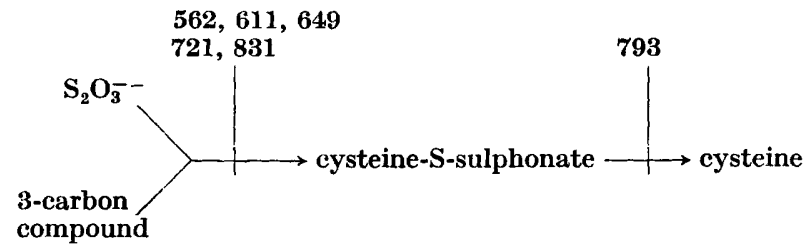

The nature of the 3-carbon compound is not yet clear, but it seems most likely that serine is the compound involved, in view of its structural relationship to cysteine-S-sulphonate. The observation reported by Hockenhull (1949) that the growth of thiosulphate-requiring mutants of Aspergillus nidulans on thiosulphate was enhanced by the addition of serine lends further support to this possibility.

\section{Assimilation of sulphide}

The results recorded in Table 1 indicate that the medium containing sodium sulphide as the sole sulphur source supported the growth of only those strains of Aspergillus nidulans which are capable of utilizing thiosulphate as sulphur source, i.e. the wild-type strain, strain 954 (group $A$ ) and strain 574 (group $B$ ). All the strains belonging to groups $C$ and $D$, on the other hand, assimilated neither sulphide nor thiosulphate. These facts appear to exclude the possibility that sulphide and thiosulphate are used by mutually independent pathways. Instead, it seems likely that these two sulphur compounds are assimilated through a common metabolic pathway.

To elucidate further the nature of sulphide utilization, experiments were carried out to examine the behaviour of heterokaryons produced between different types 
of mutants which by themselves are incapable of assimilating sulphide. The following three pairs of strains were arbitrarily chosen for this purpose:

562 ( $C$, cysteine-S-sulphonateless $) / 950(D$, methionineless);

793 ( $C$, cysteineless $) / 519(D$, methionineless $)$;

793 ( $C$, cysteineless)/721 ( $C$, cysteine-S-sulphonateless).

The results of the experiments clearly showed that all the pairs can form heterokaryons which can grow well, utilizing not only sulphate but also sulphide as sole source of sulphur. This indicates that these mutant strains acquire the ability to assimilate sulphide when their individually blocked reactions are complemented by heterokaryon formation. It is strongly suggested from these findings that sulphide, like thiosulphate, is utilized through the pathway involving cysteine-Ssulphonate, cysteine and methionine. The following scheme may therefore be presented for the pathway of sulphide utilization:

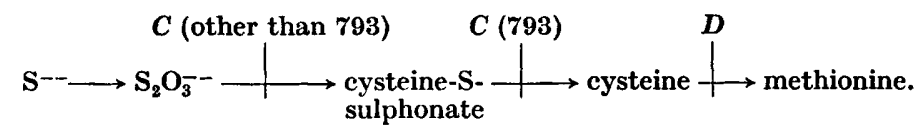

In this scheme the sequence sulphide $\rightarrow$ thiosulphate is adopted instead of thiosulphate $\rightarrow$ sulphide, since it is much more probable that thiosulphate, but not sulphide, is the immediate precursor of cysteine-S-sulphonate. It should be pointed out that the oxidative conversion of sulphide to thiosulphate has been reported to occur in mammalian tissues (Baxter, van Reen, Pearson \& Rosenberg, 1958).

If the scheme just proposed be accepted, a further problem remains to be solved: is sulphide a normal intermediate in the assimilation of sulphate? If this be so, sulphate utilization may be represented as follows:

$$
\mathrm{SO}_{4}^{--} \longrightarrow \mathrm{S}^{--} \longrightarrow \mathrm{S}_{2} \mathrm{O}_{3}^{--} \longrightarrow \text { cysteine-S-sulphonate } \longrightarrow---
$$

If, on the other hand, sulphide is not on the route from sulphate to thiosulphate, the following scheme may be more suitable to show the position of sulphide in the assimilation:

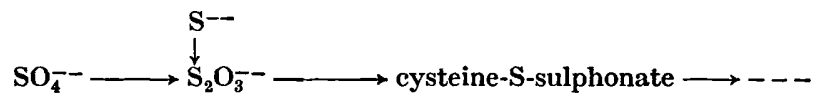

It is not yet possible from the evidence obtained in the present work to decide which is really the case. Further studies are needed to settle this point.

\section{DISCUSSION}

The results described in this paper provide evidence which indicates that in the assimilatory metabolism of Aspergillus nidulans inorganic sulphate is first reduced to sulphite and then converted to thiosulphate before its sulphur atom enters into direct combination with the carbon skeletons of sulphur-containing amino acids. It seems also fairly well established that thiosulphate reacts with a 3-carbon compound, probably serine, to form cysteine-S-sulphonate, which in turn is converted to cysteine. It may be expected that cysteine, once formed, can be either oxidized to cystine or further metabolized to methionine via the cystathionine pathway.

The mechanism by which sulphide is assimilated by Aspergillus nidulans is of interest in view of the studies reported by Schlossmann \& Lynen (1957) who showed 
that cysteine is synthesized in yeast by the direct condensation of sulphide with serine. Furthermore, Hilz et al. (1959) provided evidence that the reduction of sulphate to sulphide by yeast extracts proceeds through a pathway not involving thiosulphate as an intermediate. The data obtained in the present work suggest that sulphide is utilized by $A$. nidulans by way of thiosulphate and cysteine-Ssulphonate, although it is not certain that sulphide is an intermediate in the conversion of sulphate into thiosulphate. In any case, it seems unlikely that sulphide is the immediate precursor of cysteine in $\boldsymbol{A}$. nidulans. These conflicting data concerning the role of sulphide appear to be explicable by assuming that different pathways in the process of sulphate assimilation are operative in $\boldsymbol{A}$. nidulans as compared with yeast. The pathway functioning in Escherichia coli seems to involve sulphide as the immediate precursor of cysteine (Lampen et al. 1947) and thus resembles the pathway in yeast. The observations made by Horowitz (1950) and by Lederberg \& Tatum (1946), on the other hand, suggest that Neurospora crassa assimilates sulphate via thiosulphate and hence cysteine-S-sulphonate, as does $A$. nidulans. It may be noted that in all the previous work with $A$. nidulans (Hockenhull, 1949; Shepherd, 1956) it has been assumed that cysteine is formed from thiosulphate by way of cysteine-S-sulphonate. Although Shepherd (1956) claimed that sulphate sulphur combines with the carbon chain at the sulphite stage in the normal assimilatory metabolism, it does not seem possible to explain the results described either in the present paper or in that of Hockenhull (1949) by assuming such a pathway.

The author wishes to express his thanks to Dr Ryo Sato for his guidance and encouragements during the course of this investigation.

\section{REFERENCES}

Baxter, C. F., van Reen, R., Pearson, P. B. \& Rosenberg, C. (1958). Sulfide oxidation in rat tissues. Biochim. biophys. Acta, 27, 584.

Chapeville, F. \& Fromageot, P. (1954). La formation enzymatique de l'acide cystéine sulphinique à partir de sulfide. Biochim. biophys. Acta, 14, 415 .

Fling, M. \& Horowitz, N. H. (1951). Threonine and homoserine in extracts of a methionineless mutant of Neurospora. J. biol. Chem. 190, 277.

Fries, N. (1947). Experiments with different methods of isolating physiological mutations of filamentous fungi. Nature, Lond. 159, 199.

Hilz, H. \& Kittler, M. (1958). Enzymatische Reduktion von Sulfat zu Sulfid. Biochim. biophys. Acta, 30, 650.

Hilz, H., Kittler, M. \& Knape, G. (1959). Die Reduktion von Sulfat in der Hefe. Biochem. Z. 332, 151.

Hockenhuld, D. J. D. (1949). The sulphur metabolism of mould fungi: the use of 'biochemical mutant' strains of Aspergillus nidulans in elucidating the biosynthesis of cysteine. Biochim. biophys. Acta, 3, 326.

Horowitz, N. H. (1950). Biochemical genetics of Neurospora. Advanc. Genet. 3, 33.

KAJI, A. \& Gregory, J. D. (1959). Mechanism of sulfurylation of choline. J. biol. Chem. 234, 3007.

Kolthoff, I. M. \& Stricks, W. (1951). Polarographic investigations of reactions in aqueous solutions containing copper and cysteine (cystine). II. Reactions in ammoniacal medium in the presence and absence of sulfite. J. Amer. chem. Soc. 73, 1728.

Lampen, J. O., Roepke, R. R. \& Jones, M. J. (1947). Studies on the sulfur metabolism of Escherichia coli. III. Mutant strains of Escherichia coli unable to utilize sulfate for their complete sulfur requirements. Arch. Biochem. 13, 55. 
Lederberg, J. \& TAtum, E. L. (1946). Detection of biochemical mutants of microorganisms. J. biol. Chem. 165, 381.

LezIUs, A. (1959). Thiosulfardesulfhydrase und Sulfitreduktase, zwei Enzyme des Schwefelstoffwechsels der Bäckerhefe. Dissertation, Universität zu München.

Nakamura, T. \& Sato, R. (1960). Cysteine-S-sulphonate as an intermediate in microbial synthesis of cysteine. Nature, Lond. 185, 163.

Ponteconvo, G. (1947). Genetic systems based on heterokaryosis. Cold Spr. Harb. Symp. quant. Biol. 11, 193.

Ponteconvo, G. (1953). The genetics of Aspergillus nidulans. Advanc. Genet. 5, 141.

Pontecorvo, G. \& Sermonti, G. (1954). Parasexual recombination in Penicillium chrysogenum. J. gen. Microbiol. 11, 94.

Ponteconvo, G., Gloor, E. T. \& Forbes, E. (1954). Analysis of mitotic recombination in Aspergillus nidulans. J. Genet. 52, 226.

Ragland, J. B. (1959). The role of ATP-sulfurylase in the biosynthesis of cysteine and methionine by Neurospora. Arch. Biochem. Biophys. 84, 541.

Schlossmann, K. \& Lynen, F. (1957). Biosynthese des Cysteins aus Serin und Schwefelwasserstoff. Biochem. Z. 328, 591.

Schmidt, E. \& Wagner, W. (1904). Über Cholin, Neurin und verwandte Verbindungen. II. Cholin. Liebigs Ann. 337, 51.

Shepherd, C. J. (1956). Pathway of cysteine synthesis in Aspergillus nidulans. J. gen. Microbiol. 15, 29.

Spencer, B. \& Harada, T. (1960). The role of choline sulphate in the sulphur metabolism of fungi. Biochem. J. 77, 305.

Teas, H. J., Honowitz, N. H. \& Fling, M. (1948). Homoserine as a precursor of threonine and methionine in Neurospora. J. biol. Chem. 172, 651.

Wruson, L. G. \& Bandurski, R. S. (1958). Enzymatic reduction of sulfate. J. Amer. chem. Soc. 80, 5576. 